\title{
Declines in serum CYFRA21-1 and carcinoembryonic antigen as predictors of chemotherapy response and survival in patients with advanced non-small cell lung cancer
}

\author{
LIANG YANG ${ }^{1}, \mathrm{XIN} \mathrm{CHEN}^{1}, \mathrm{YUE} \mathrm{LI}^{2}, \mathrm{JUN}^{\mathrm{Y} A N G}{ }^{1}$ and $\mathrm{LI} \mathrm{TANG}^{1}$ \\ ${ }^{1}$ Department of Echocardiography, The First Affiliated Hospital of China Medical University, Shenyang 110001; \\ ${ }^{2}$ Department of Laboratory Medicine, Fengtian Hospital of Shenyang Medical College, Shenyang 110001, P.R. China
}

Received October 18, 2011; Accepted November 28, 2011

DOI: $10.3892 /$ etm.2012.570

\begin{abstract}
The aim of this study was to investigate the clinical value of serum cytokeratin 19 fragment (CYFRA21-1) and carcinoembryonic antigen (CEA) in the prediction of chemotherapy response and prognosis in patients with advanced non-small cell lung cancer (NSCLC). Serum CYFRA21-1 and CEA levels of 98 patients with advanced NSCLC were measured using immunoradiometric kits prior to and after 2 cycles of chemotherapy. After 2 cycles of chemotherapy, 45 patients achieved a radiological objective response (OR), 30 patients achieved stable disease (SD) and 23 patients had progressive disease (PD). Serum CYFRA21-1 and CEA were significantly decreased compared to baseline levels $(\mathrm{P}<0.001)$. By ROC curve analysis, a $\geq 60 \%$ reduction in CYFRA21-1 and a $\geq 25 \%$ reduction in CEA were the optimal cut-off levels with best sensitivity and specificity for the diagnosis of radiologic OR. The median survival of all patients was 10.2 months (range 2.6-26.3). Univariate survival analysis showed that the Eastern Cooperative Oncology Group (ECOG) performance status (PS) score, radiologic OR, $a \geq 60 \%$ reduction in CYFRA $21-1$ and $a \geq 25 \%$ reduction in CEA were significant prognostic factors for better overall survival. The median overall survival time in patients with a $\geq 60 \%$ reduction in CYFRA21-1 was significantly longer than in those with a $<60 \%$ reduction $(\mathrm{P}<0.001)$. Similarly, the median overall survival time in patients with a $\geq 25 \%$ reduction in CEA was also significantly longer than in those with a $<25 \%$ reduction $(\mathrm{P}<0.001)$. Multivariate analysis showed that ECOG PS score, a $\geq 60 \%$ reduction in CYFRA21-1 and a $\geq 25 \%$ reduction in CEA were independent prognostic factors
\end{abstract}

Correspondence to: Dr Xin Chen, Department of Echocardiography, The First Affiliated Hospital of China Medical University, Shenyang 110001, P.R. China

E-mail: cmu_cx@126.com

Key words: cytokeratin 19 fragment, carcinoembryonic antigen, non-small cell lung cancer, chemotherapy response, prognosis of survival, while radiologic OR was not. In conclusion, a $\geq 60 \%$ reduction in CYFRA $21-1$ and a $\geq 25 \%$ reduction in CEA may be reliable surrogate markers for the prediction of chemothrapy response and prognosis, especially for the diagnosis of radiologic OR.

\section{Introduction}

Lung cancer is the leading cause of cancer-related death in the world (1). In 2010, an estimated 222,520 new cases and 157,300 deaths were anticipated in the US (2). Non-small cell lung cancer (NSCLC) accounts for more than $85 \%$ of all cases of lung cancer (3). Approximately $40 \%$ of patients with NSCLC present with advanced-stage disease at the time of diagnosis (3). The standard treatment for these patients is systemic chemotherapy, which improves both quality of life and survival (4). Until recently, platinum- or non-platinumbased, two-drug regimens were considered the standard of care for advanced NSCLC patients $(4,5)$. However, the vast majority of patients with advanced NSCLC failed to benefit from combined chemotherapy (6). The WHO and Response Evaluation Criteria in Solid Tumors (RECIST) criterias, based on radiologic detections, were used to assess objective response after combined chemotherapy (7). Usually, the objective response (OR) of unmeasurable lesions, such as for atelectasis, pericardial effusion, pleural effusion, lymphatic vessel invasion and pleural-type tumors are difficult to evaluate using radiologic results (8). Moreover, a decrease in tumor volume as determined by radiologic images cannot accurately predict the survival of patients with advanced NSCLC (9). Therefore, more effective and feasible markers are required for the prediction of chemotherapy response and prognosis in patients with advanced NSCLC. Serum tumor markers, as a potential and more effective method to determine chemotherapy response and predict prognosis, have been studied extensively in the past. It has been proven that cytokeratin 19 fragment (CYFRA21-1) and carcinoembryonic antigen (CEA) may be useful predictive factors of chemotherapy response and prognosis in advanced NSCLC patients. The aim of this study was to investigate the clinical value of serum CYFRA21-1 and CEA in the prediction of chemotherapy response and prognosis in patients with advanced NSCLC. 


\section{Materials and methods}

Patient inclusion criteria. To be eligible for inclusion in this study, the following criteria were established: i) patients had a histological or cytological confirmation of clinical stage IIIB or IV NSCLC; ii) at least one measurable lesion; iii) patients were able to withstand at least 2 cycles of first-line platinum-based combined chemotherapy; iv) Eastern Cooperative Oncology Group (ECOG) performance status (PS) 0-2; v) no main organ dysfunction and hematopoietic function, normal liver and renal function, without any serious complications.

Assessment criteria of objective response. Assessments of the objective response (OR) were based on WHO and RECIST criteria (7), including complete response (CR), partial response (PR), stable disease (SD) or progressive disease (PD). OR was defined as CR plus PR, and no response (NR) was defined as $\mathrm{SD}$ plus PD. OR was evaluated and a confirmative chest computed tomography (CT) scan was performed after every 2 cycles of chemotherapy.

Assessment criteria of serum markers. Two serum samples from untreated lung cancer patients were prospectively collected: the first prior to the first cycle of chemotherapy, and the second after the second cycle of chemotherapy. All samples were frozen and stored at $-80^{\circ} \mathrm{C}$. All assays were performed using commercial kits: CYFRA21-1 and CEA (ELSA; CIS Biointernational, France) (10), with investigators blinded to clinical information. The cut-off value of CYFRA21-1 and CEA was 3.2 and $3.4 \mathrm{ng} / \mathrm{ml}$, respectively. Patients were defined as assessable when at least one serum level of either CYFRA21-1 or CEA was above the normal cut-off values.

Statistical analysis. Statistical analysis was performed using SPSS version 17.0 software (SPSS Inc., Chicago, IL, USA). Comparisons of serum CYFRA21-1 or CEA prior to and after 2 cycles of chemotherapy were analyzed by Wilcoxon's signed rank test. Associations between categorical variables were evaluated using the $\chi^{2}$-test. The ROC curve was used to assess the optimal cut-off levels of the declines of serum CYFRA21-1 and CEA in the diagnosis of radiologic OR. The Youden index was used to identify the optimal cut-off levels.

All survival data were updated in May 1, 2011. Overall survival (OS) was calculated from the initiation of chemotherapy until death. Time to progression (TTP) was calculated from the date of registration to progression or last contact. Survival curves were generated with the Kaplan-Meier method and compared by the log-rank test and generalized Wilcoxon's test. Meanwhile, the multivariate survival analysis was performed to investigate the independent prognostic factors using Cox proportional hazards regression model. All tests were two-sided, and a value of $\mathrm{P}<0.05$ was considered statistically significant.

\section{Results}

Baseline characteristics of patients. Between May 2006 and May 2010, a total of 98 patients with advanced NSCLC who were admitted to The First Affiliated Hospital of China Medical University, were enrolled in this study. The median age was 58 years (range 27-84), including 65 males and
Table I. Baseline characteristics of the NSCLC patients.

\begin{tabular}{|c|c|}
\hline Characteristics & No. of patients (\%) \\
\hline \multicolumn{2}{|l|}{ Gender } \\
\hline Male & $65(66.3)$ \\
\hline Female & $33(33.7)$ \\
\hline \multicolumn{2}{|l|}{ Age (years) } \\
\hline Median (range) & $58(27-84)$ \\
\hline$\leq 65$ & $60(61.2)$ \\
\hline$>65$ & $38(38.8)$ \\
\hline \multicolumn{2}{|l|}{ Histology } \\
\hline Squamous cell carcinoma & $28(28.6)$ \\
\hline Adenocarcinoma & $61(62.2)$ \\
\hline Adenosquamous carcinomas & $9 \quad(9.2)$ \\
\hline \multicolumn{2}{|l|}{ Clinical stage } \\
\hline III & $53(54.1)$ \\
\hline IV & $45(45.9)$ \\
\hline \multicolumn{2}{|l|}{ ECOG PS score } \\
\hline $0-1$ & $54(55.1)$ \\
\hline 2 & $44(44.9)$ \\
\hline \multicolumn{2}{|l|}{ CYFRA21-1 baseline (ng/ml) } \\
\hline Median value (range) & $6.4(1.5-144.7)$ \\
\hline Normal $(\leq 3.2)$ & $22(22.4)$ \\
\hline Abnormal $(>3.2)$ & $76(77.6)$ \\
\hline \multicolumn{2}{|l|}{ CEA baseline (ng/ml) } \\
\hline Median value (range) & $13.9(1.1-985.1)$ \\
\hline Normal $(\leq 3.4)$ & $16(16)$ \\
\hline Abnormal (>3.4) & $82(82)$ \\
\hline \multicolumn{2}{|l|}{ Chemotherapy response } \\
\hline $\mathrm{CR}$ & $1(1.0)$ \\
\hline PR & $44(44.9)$ \\
\hline SD & $30(30.6)$ \\
\hline PD & $23(23.5)$ \\
\hline \multicolumn{2}{|l|}{ Last follow-up status } \\
\hline Alive & $19(19.4)$ \\
\hline Dead & 79 (80.6) \\
\hline
\end{tabular}

CYFRA21-1, cytokeratin 19 fragment; CEA, carcinoembryonic antigen; ECOG, Eastern Cooperative Oncology Group; PS, performance status; CR, complete response; PR, partial response; SD, stable disease; $P D$, progressive disease.

33 females. There were 61 patients with adenocarcinomas, 28 with squamous carcinomas and 9 with adenosquamous carcinomas. According to the TNM staging system for lung cancer by the 6th edition of the International Union Against Cancer (UICC) and the American Joint Committee on Cancer (AJCC) (11), the study included 53 patients with stage IIIB and 45 patients with stage IV. There were 54 patients with a ECOG PS score of 0-1. All patients received a median of 4 cycles of chemotherapy (range 2-6). Baseline characteristics of the patients are shown in Table I.

Association between chemotherapy response and decreases in the serum markers. Among 98 patients with advanced NSCLC, $45.9 \%$ (45/98) achieved OR after 2 cycles of chemotherapy, 

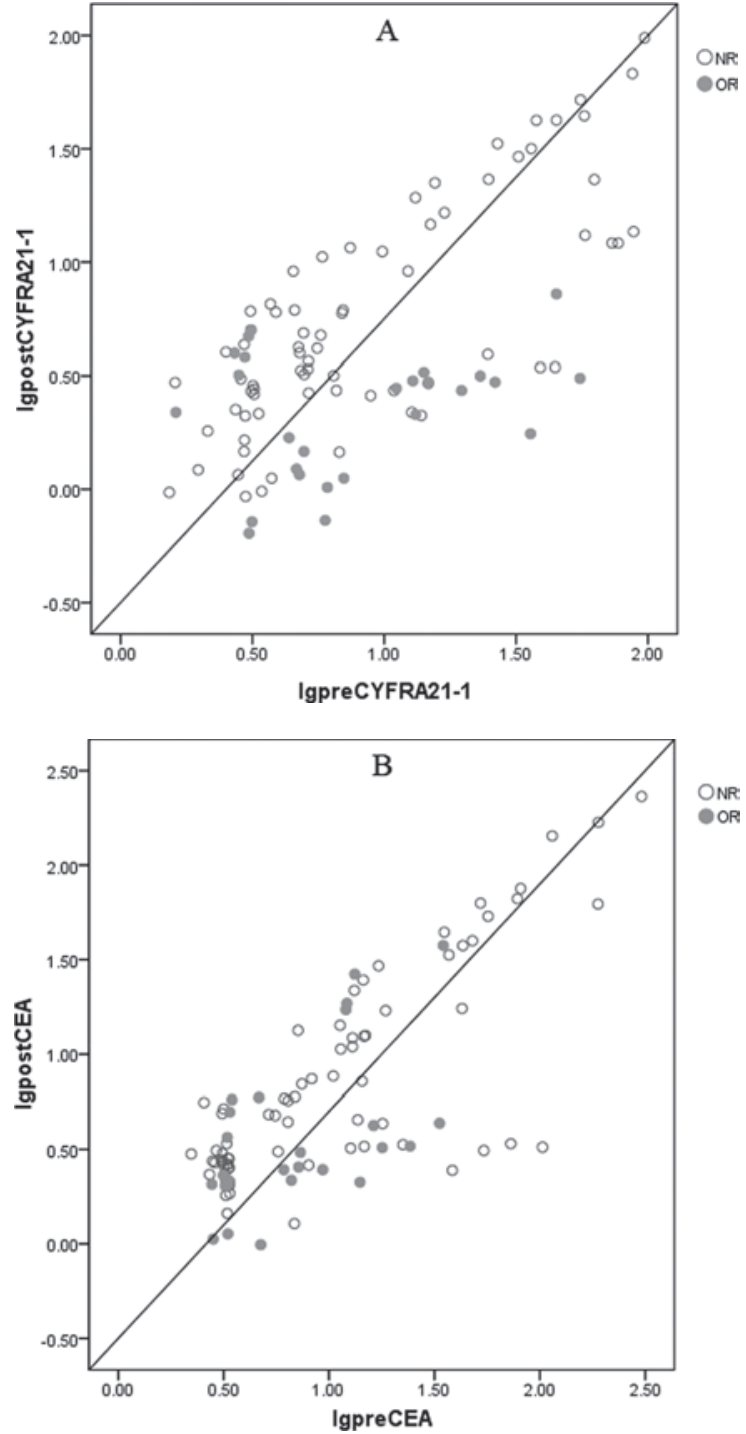

Figure 1. (A) CYFRA21-1 and (B) CEA natural logarithm prior to and after 2 cycles of chemotherapy according to radiologic objective response (OR). NR, no response.

including 1 patient with CR, 44 patients with PR, 30 patients with SD and 23 patients with PD. The median values of serum CYFRA21-1 prior to and after chemotherapy were $6.4 \mathrm{ng} / \mathrm{ml}$ (range 1.5-144.7) and $3.4 \mathrm{ng} / \mathrm{ml}$ (range 0.6-97.7), respectively. The median values of serum CEA prior to and after chemotherapy were $13.9 \mathrm{ng} / \mathrm{ml}$ (range 1.1-985.1) and $3.9 \mathrm{ng} / \mathrm{ml}$ (range 1.0-375.5), respectively. After 2 cycles of chemotherapy, serum CYFRA21-1 and CEA were significantly decreased compared to baseline levels $(\mathrm{P}<0.0001)$.

As shown in Fig. 1, the values in the horizontal axis represent serum CYFRA21-1 and CEA prior to chemotherapy, and those in the vertical axis represent serum CYFRA21-1 and CEA after 2 cycles of chemotherapy. This reflected the relationship between serum CYFRA21-1 or CEA and radiologic OR. If there was no significant difference in serum CYFRA21-1 or CEA prior to and after 2 cycles of chemotherapy, the corresponding points should fall along the straight line. In fact, most of the data points were below the line, especially for those of serum CYFRA21-1, suggesting that chemotherapy induced declines in serum CYFRA21-1 and/or CEA in the majority of patients.
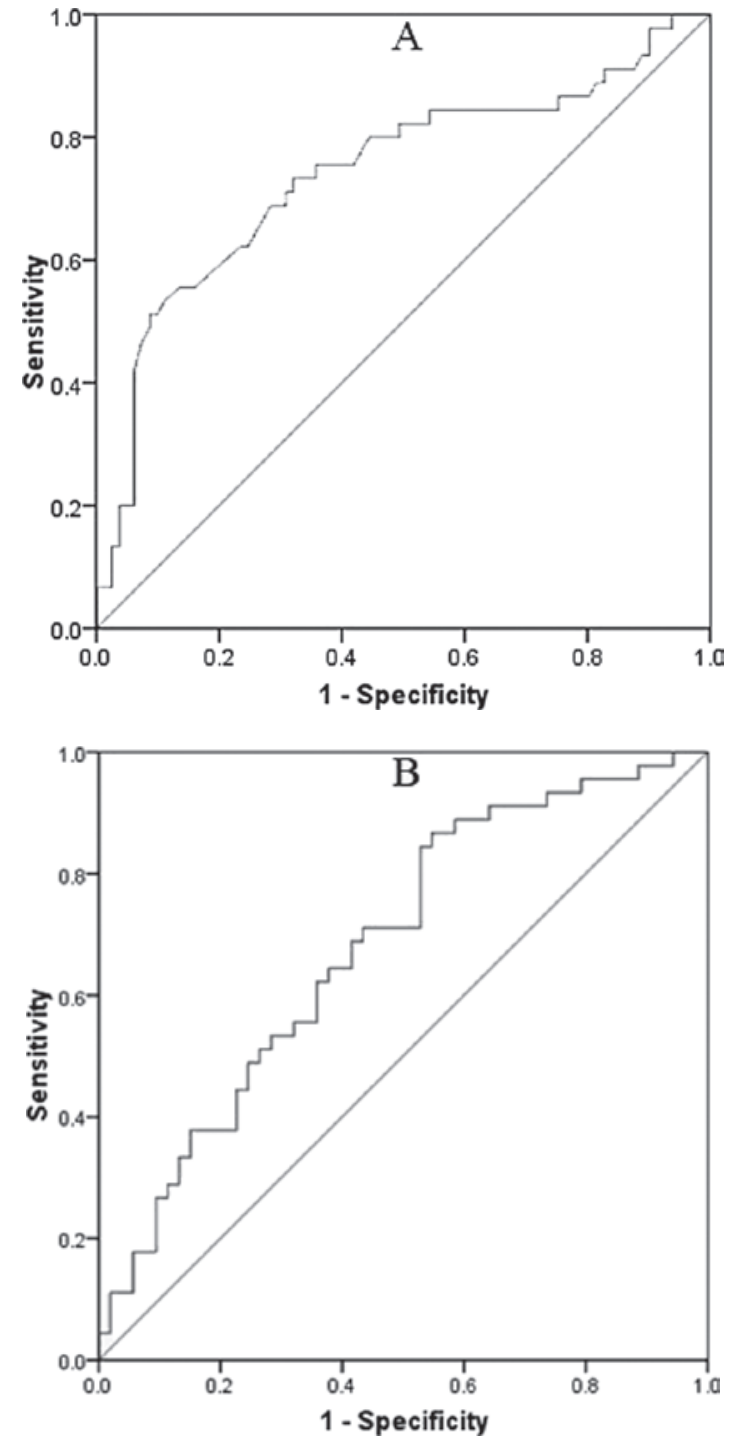

Figure 2. ROC curves analysis of serum (A) CYFRA21-1 and (B) CEA in the diagnosis of radiologic OR after 2 cycles of chemotherapy.

ROC curves of serum CYFRA21-1 and CEA. Analysis of the ROC curves was carried out to assess the correlation between declines in serum CYFRA21-1 or CEA and radiologic OR after 2 cycles of chemotherapy (Fig. 2). The area under the ROC curve (AUC) was 0.727 (95\% CI 0.600-0.857) for CYFRA21-1 and 0.629 (95\% CI 0.48-0.771) for CEA. After 2 cycles of chemotherapy, $a \geq 60 \%$ reduction in CYFRA21-1 and $a \geq 25 \%$ reduction in CEA were the optimal cut-off levels with best sensitivity and specificity for the diagnosis of radiologic OR. When there was a $\geq 60 \%$ reduction in serum CYFRA21-1, the sensitivity and specificity values were 77.9 and $78.2 \%$, respectively. When there was a $\geq 25 \%$ reduction in serum CEA, the sensitivity and specificity were 70.5 and $68.7 \%$, respectively. Therefore, a $\geq 60 \%$ reduction in CYFRA21-1 and $\mathrm{a} \geq 25 \%$ reduction in CEA were defined as 'serum marker response'.

Univariate and multivariate survival analysis. During the study period, 79 of $98(80.6 \%)$ patients with advanced NSCLC died. The median survival of all patients was 10.2 months (range 2.6-26.3). As shown in Fig. 3, the median OS time in 

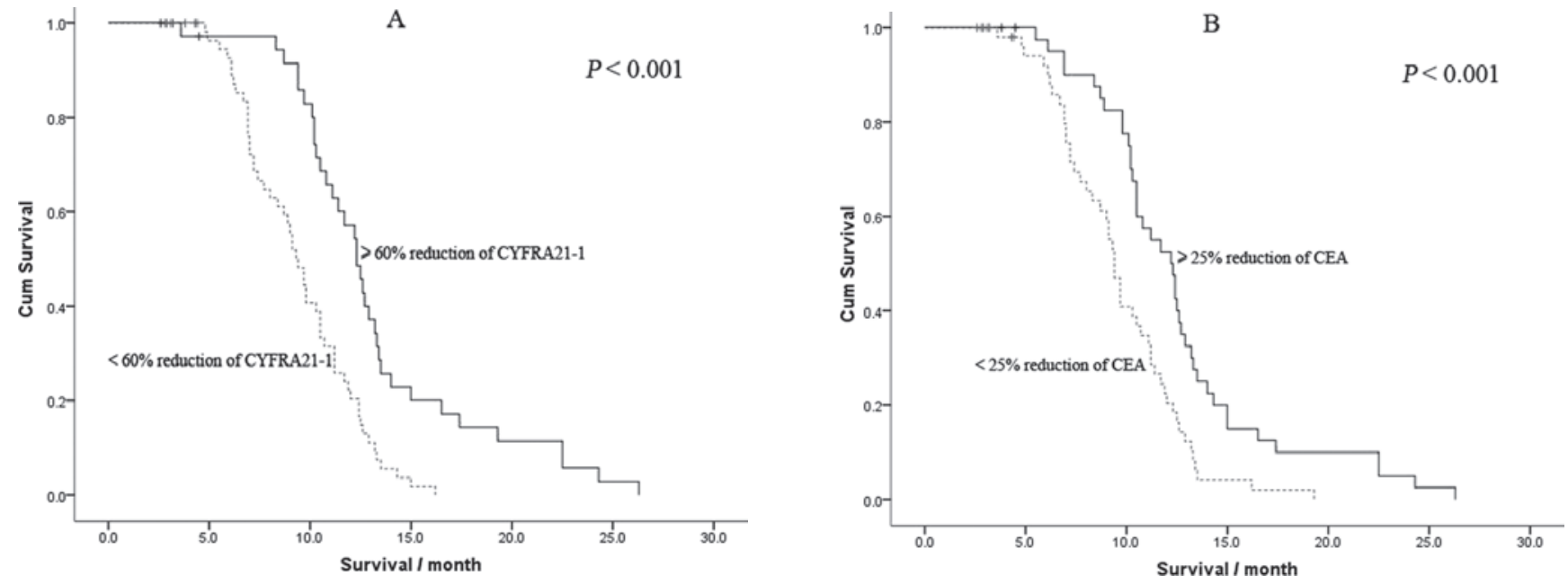

Figure 3. Overall survival curve in patients with different serum (A) CYFRA21-1 and (B) CEA responses.

Table II. Univariate survival analysis.

\begin{tabular}{|c|c|c|c|c|}
\hline Prognostic factors & No. of patients & MST (months) & $95 \% \mathrm{CI}$ & P-value \\
\hline Age (years) & & & & 0.504 \\
\hline$\leq 65$ & 60 & 10.1 & $9.4-10.8$ & \\
\hline$>65$ & 38 & 11.3 & $9.9-12.5$ & \\
\hline Gender & & & & 0.170 \\
\hline Male & 65 & 11.2 & $10.3-12.1$ & \\
\hline Female & 33 & 10.2 & 9.3-10.1 & \\
\hline Clinical stage & & & & 0.761 \\
\hline III & 37 & 10.6 & $9.1-10.3$ & \\
\hline IV & 61 & 10.9 & $9.7-11.3$ & \\
\hline ECOG PS score & & & & 0.001 \\
\hline 0 & 59 & 11.7 & $9.9-12.3$ & \\
\hline $1-2$ & 39 & 9.6 & $8.3-10.9$ & \\
\hline Histology & & & & 0.088 \\
\hline Squamous cell carcinoma & 28 & 11.2 & $9.5-12.9$ & \\
\hline Adenocarcinoma & 61 & 9.7 & $9.0-10.4$ & \\
\hline Adenosquamous carcinomas & 9 & 10.4 & $9.2-11.1$ & \\
\hline Radiologic OR & & & & 0.034 \\
\hline Yes & 55 & 11.3 & 10.4-13.0 & \\
\hline No & 53 & 9.8 & $9.0-10.6$ & \\
\hline CYFRA21-1 baseline level & & & & 0.401 \\
\hline Normal & 22 & 10.7 & $9.1-10.5$ & \\
\hline$>3.2 \mathrm{ng} / \mathrm{ml}$ & 76 & 11.1 & $9.4-11.6$ & \\
\hline CEA baseline level & & & & 0.683 \\
\hline Normal & 16 & 10.7 & $9.7-12.7$ & \\
\hline$>3.4 \mathrm{ng} / \mathrm{ml}$ & 82 & 10.2 & $9.5-10.5$ & \\
\hline$\geq 60 \%$ reduction in CYFRA $21-1$ & & & & $<0.001$ \\
\hline Yes & 36 & 11.6 & $11.5-12.9$ & \\
\hline No & 62 & 9.3 & $8.8-9.8$ & \\
\hline$\geq 25 \%$ reduction in CEA & & & & $<0.001$ \\
\hline Yes & 40 & 11.2 & 10.9-13.1 & \\
\hline No & 58 & 8.9 & $9.1-9.9$ & \\
\hline
\end{tabular}

MST, median survival time; CI, confidence interval; ECOG, Eastern Cooperative Oncology Group; PS, performance status; CYFRA21-1, cytokeratin 19 fragment; CEA, carcinoembryonic antigen; OR, objective response. 
Table III. Multivariate survival analysis.

\begin{tabular}{lccc}
\hline Prognostic factors & HR & $95 \%$ CI & P-value \\
\hline $\begin{array}{l}\text { ECOG PS } \\
0\end{array}$ & 1.000 & & $<0.0001$ \\
$\quad 1-2$ & 2.904 & $1.763-4.784$ & \\
Radiologic OR & & & 0.1910 \\
$\quad$ Yes & 1.000 & & \\
$\quad$ No & 1.563 & $0.807-2.924$ & \\
$\geq 60 \%$ reduction in & & & 0.0010 \\
CYFRA21-1 & & & \\
$\quad$ No & 1.000 & & 0.0380 \\
$\quad$ Yes & 0.254 & $0.110-0.588$ & \\
$\geq 25 \%$ reduction in CEA & & & \\
No & 1.000 & & \\
$\quad$ Yes & 0.417 & $0.182-0.954$ & \\
\hline
\end{tabular}

HR, hazards ratio; CI, confidence interval, ECOG, Eastern Cooperative Oncology Group; PS, performance status; OR, objective response. CYFRA21-1, cytokeratin 19 fragment; CEA, carcinoembryonic antigen.

patients with a $\geq 60 \%$ reduction in CYFRA21-1 was significantly longer compared to those with a $<60 \%$ reduction (11.6 vs. 9.3 months, $\mathrm{P}<0.001)$. In addition, the median OS time in patients with a $\geq 25 \%$ reduction in CEA was also significantly longer compared to those with a $<25 \%$ reduction (11.2 vs. 8.9 months, $\mathrm{P}<0.001)$.

Univariate survival analysis showed that ECOG PS score, radiologic OR, a $\geq 60 \%$ reduction in CYFRA21-1 and $\mathrm{a} \geq 25 \%$ reduction in CEA were significant prognostic factors for better OS (Table II). However, age, gender, clinical stage, histological type and baseline levels of CYFRA21-1 and CEA were not related to prognosis. Results from the Cox regression analysis are shown in Table III. In this analysis, the independent prognostic roles of ECOG PS score, a $\geq 60 \%$ reduction in CYFRA21-1 and a $\geq 25 \%$ reduction in CEA were confirmed, while radiologic OR was not an independent prognostic factor.

\section{Discussion}

CYFRA21-1 is an acidic protein of $40 \mathrm{kDa}$ that is part of the cytoskeleton of epithelial cells (12). CYFRA21-1 is a specific and reproducible negative-prognostic marker for NSCLC (13). Many studies have confirmed that CYFRA21-1 is both a sensitive and specific tumor marker for NSCLC and especially for squamous cell carcinoma (14). It appears more sensitive and more specific than other tumor markers, such as CEA and NSE, and slightly better than squamous cell carcinoma-antigen (SCC) in squamous cell carcinoma (15). CEA is a glycoprotein expressed during early fetal life, and is the product of the CEACAM5-gen (16). CEA is an oncofetal protein attached to epithelial cell apical membrane via its C-terminal glycosylphosphatidylinositol anchor, a member of the immunoglobulin superfamily of cell adhesion molecules (17). Usually, CEA is overexpressed in a variety of neoplasms, such as colorectal, breast, bladder, gastric, pancreatic and lung carcinomas (16). CEA is a good monitoring marker for conventional chemotherapy. High serum CEA levels have been associated with disease progression and relapse in patients with advanced NSCLC (18). Several reports have been published concerning the prognostic value of serum tumor markers in patients with advanced NSCLC, for example CYFRA21-1, CEA, NSE and SCC (19). However, no reports concerning the relationship of declines in serum CYFRA21-1 or CEA with chemotheapy response and prognosis in patients with advanced NSCLC have been previously published. To our knowledge, this study is the first to propose that a $\geq 60 \%$ reduction in CYFRA21-1 and $\mathrm{a} \geq 25 \%$ reduction in CEA after 2 cycles of chemotherapy can be regarded as possible surrogate markers of chemotherapy response and prognosis in patients with advanced NSCLC.

In the present study, we found that decreases in serum CYFRA21-1 and CEA prior to and after 2 cycles of chemotherapy were correlated with chemotherapy response. We also found that $\mathrm{a} \geq 60 \%$ reduction in CYFRA21-1 and $\mathrm{a} \geq 25 \%$ reduction in CEA after 2 cycles of chemotherapy were independent prognostic factors for patients with advanced NSCLC in multivariate survival analysis, while radiologic OR was not an independent prognostic factor. Nisman et al (20) found that there was no correlation between radiologic OR and survival, while declines in serum CYFRA21-1 after 2 cycles of chemotherapy were closely related to survival. Ardizzoni et al (21) studied 107 patients with advanced NSCLC and also observed that declines in serum CYFRA21-1 and CEA were closely related to chemotherapy response and survival, whereas radiologic OR had no correlation with survival. Similar to previous studies, our study also demonstrated that the declines in serum CYFRA21-1 and CEA were closely related to chemotherapy response and survival, especially related to radiologic OR. After 2 cycles of chemotherapy, a $\geq 60 \%$ reduction in CYFRA21-1 and $\mathrm{a} \geq 25 \%$ reduction in CEA were the optimal cut-off levels, with best sensitivity and specificity for the diagnosis of radiologic OR. Univariate survival analysis showed that ECOG PS score, radiologic OR, a $\geq 60 \%$ reduction in CYFRA21-1 and a $\geq 25 \%$ reduction in CEA were significant prognostic factors. After 2 cycles of chemotherapy, the median OS time in patients with a $\geq 60 \%$ reduction in CYFRA21-1 was significantly longer compared to those with a $<60 \%$ reduction. Similarly, the median OS time in patients with a $\geq 25 \%$ reduction in CEA was also significantly longer compared to those with a $<25 \%$ reduction. Multivariate analysis further confirmed the clinical value of declines in serum CYFRA21-1 and CEA in the prediction of chemotherapy response and prognosis in patients with advanced NSCLC. In multivariate analysis, the independent prognostic roles of ECOG PS score, $\geq 60 \%$ reduction in CYFRA21- 1 and $\geq 25 \%$ reduction in CEA were confirmed, while radiologic OR was not an independent prognostic factor.

In conclusion, our study demonstrated that a $\geq 60 \%$ reduction in CYFRA21-1 and a $\geq 25 \%$ reduction in CEA may be reliable surrogate markers for the prediction of chemotherapy response and prognosis, particularly for the diagnosis of radiologic OR. Due to a limitation in the sample of patients, this conclusion should be further confirmed by large case-control studies with an adequate methodological quality and properly controlled for possible confounds. 


\section{Acknowledgements}

The authors would like to thank Jia-Li Liu (Department of Oncology, The First People's Hospital, Yueyang, Hunan) for his valuable contribution and revising of the manuscript.

\section{References}

1. Jemal A, Murray T, Samuels A, Ghafoor A, Ward E and Thun MJ: Cancer statistics, 2003. CA Cancer J Clin 53: 5-26, 2003.

2. Jemal A, Siegel R, Xu J and Ward E: Cancer statistics, 2010. CA Cancer J Clin 60: 277-300, 2010.

3. Lovly CM and Carbone DP: Lung cancer in 2010: one size does not fit all. Nat Rev Clin Oncol 8: 68-70, 2011.

4. Ramalingam SS, Dahlberg SE, Langer CJ, Gray R, Belani CP, Brahmer JR, Sandler AB, Schiller JH, Johnson DH and Eastern Cooperative Oncology Group: Outcomes for elderly, advanced-stage non small-cell lung cancer patients treated with bevacizumab in combination with carboplatin and paclitaxel: analysis of Eastern Cooperative Oncology Group Trial 4599. J Clin Oncol 26: 60-65, 2008.

5. Pujola JL, Barlesia F and Daurèsa JP: Should chemotherapy combinations for advanced non-small cell lung cancer be platinum-based? A meta-analysis of phase III randomized trials. Lung Cancer 3: 335-345, 2006.

6. Lilenbaum RC, Langenberg P and Dickersin K: Single agent versus combination chemotherapy in patients with advanced non-small cell lung carcinoma: a meta-analysis of response, toxicity, and survival. Cancer 82: 116-126, 1998.

7. Nishino M, Jackman DM, Hatabu H, Yeap BY, Cioffredi LA, Yap JT, Jänne PA, Johnson BE and Van den Abbeele AD: New Response Evaluation Criteria in Solid Tumors (RECIST) guidelines for advanced non-small cell lung cancer: comparison with original RECIST and impact on assessment of tumor response to targeted therapy. AJR Am J Roentgenol 195: W221-W228, 2010.

8. Jin B, Huang AM, Zhong RB and Han BH: The value of tumor markers in evaluating chemotherapy response and prognosis in Chinese patients with advanced non-small cell lung cancer. Chemotherapy 56: 417-423, 2010.

9. Birchard KR, Hoang JK, Herndon JE Jr and Patz EF Jr: Early changes in tumor size in patients treated for advanced stage non-small cell lung cancer do not correlate with survival. Cancer 115: 581-586, 2009.
10. Buccheri G and Ferrigno D: Cytokeratin-derived markers of lung cancer. Expert Rev Mol Diagn 1: 315-322, 2001.

11. Rami-Porta R, Crowley JJ and Goldstraw P: The revised TNM staging system for lung cancer. Ann Thorac Cardiovasc Surg 15: $4-9,2009$.

12. Hanagiri T, Sugaya M, Takenaka M, Oka S, Baba T, Shigematsu Y, Nagata Y, Shimokawa H, Uramoto H, Takenoyama M, Yasumoto K and Tanaka F: Preoperative CYFRA 21-1 and CEA as prognostic factors in patients with stage I non-small cell lung cancer. Lung Cancer 74: 112-117, 2011.

13. Ando S, Suzuki M, Yamamoto N, Iida T and Kimura H: The prognostic value of both neuron-specific enolase (NSE) and Cyfra21-1 in small cell lung cancer. Anticancer Res 24: 1941-1946, 2004.

14. Sung HJ and Cho JY: Biomarkers for the lung cancer diagnosis and their advances in proteomics. BMB Rep 41: 615-625, 2008.

15. Barak V, Goike H, Panaretakis KW and Einarsson R: Clinical utility of cytokeratins as tumor markers. Clin Biochem 37: 529-540, 2004.

16. Arrieta O, Saavedra-Perez D, Kuri R, Aviles-Salas A, Martinez L, Mendoza-Posada D, Castillo P, Astorga A, Guzman E and de la Garza J: Brain metastasis development and poor survival associated with carcinoembryonic antigen (CEA) level in advanced non-small cell lung cancer: a prospective analysis. BMC Cancer 9: 119, 2009.

17. Horst AK and Wagener C: CEA-related CAMs. Handb Exp Pharmacol 165: 283-341, 2004.

18. Wang CY, Huang MS, Huang MH, Lee HC and Hsu HS: Persistently high serum carcinoembryonic antigen levels after surgery indicate poor prognosis in patients with stage I nonsmall-cell lung cancer. J Surg Res 163: e45-e50, 2010.

19. Cho WC: Potentially useful biomarkers for the diagnosis, treatment and prognosis of lung cancer. Biomed Pharmacother 61: 515-519, 2007.

20. Nisman B, Biran H, Heching N, Barak V, Ramu N, Nemirovsky I and Peretz T: Prognostic role of serum cytokeratin 19 fragments in advanced non-small-cell lung cancer: association of marker changes after two chemotherapy cycles with different measures of clinical response and survival. Br J Cancer 98: 77-79, 2008.

21. Ardizzoni A, Cafferata MA, Tiseo M, Filiberti R, Marroni P, Grossi $F$ and Paganuzzi M: Decline in serum carcinoembryonic antigen and cytokeratin 19 fragment during chemotherapy predicts objective response and survival in patients with advanced non-small cell lung cancer. Cancer 107: 2842-2849, 2006. 